\title{
DIFFERENTIABILITY OF WEAK SOLUTIONS TO AN ABSTRACT INHOMOGENEOUS DIFFERENTIAL EQUATION
}

\author{
C. C. TRAVIS ${ }^{1}$
}

\begin{abstract}
A characterization is given of those strongly continuous semigroups $T(t)$ for which the function $\int_{0}^{t} T(t-s) f(s) d s$ is continuously differentiable on $t>0$ for each continuous $f:[0, \infty) \rightarrow X$.
\end{abstract}

1. Introduction. Let $X$ be a Banach space and consider the Cauchy problem

$$
\begin{gathered}
d u(t) / d t=A u(t)+f(t) \text { on }[0, r], \\
u(0)=x \in D(A),
\end{gathered}
$$

where $f$ is a continuous $X$-valued function on $[0, \infty)$ and $A$ is the infinitesimal generator of a strongly continuous semigroup $T(t), t>0$, of bounded linear operators in $X$. A function $u$ is said to be a strong solution of (1.1) if $u$ is continuously differentiable, $u(t) \in D(A)$ for $t \in[0, r]$, and $u$ satisfies equation (1.1) for $t \in[0, r]$. A weak solution of (1.1) is a continuous function $u$ with the property that for every $h \in D\left(A^{*}\right)$ the function $\langle u(t), h\rangle$ is absolutely continuous and satisfies

$$
(d / d t)\langle u(t), h\rangle=\left\langle u(t), A^{*} h\right\rangle+\langle f(t), h\rangle, \quad u(0)=x,
$$

almost everywhere on $[0, r]$. J. Ball [2] has shown that $(1.1)$ has the unique weak solution $v$ given by

$$
v(t)=T(t) x+\int_{0}^{t} T(t-s) f(s) d s .
$$

It is well known, however, that without some restrictions on the semigroup $T(t)$, $t>0$, or the continuous function $f$, a weak solution of equation (1.1) need not be a strong solution. R. Phillips [7] has shown that if $x \in D(A)$ and $f$ is continuously differentiable, then the weak solution (1.3) is the unique strong solution of (1.1). The condition that $f$ be continuously differentiable can be weakened by requiring further conditions on the strongly continuous semigroup $T(t), t>0$. For example, it is known [5] that (1.3) is a strong solution of (1.1) for every Hölder continuous function $f$ if $T(t), t \geqslant 0$, is holomorphic.

Our objective in this paper is to characterize the class of strongly continuous semigroups for which a weak solution of equation (1.1) is a strong solution when $f \in C([0, r] ; X)$. We demonstrate that (1.3) is a strong solution of (1.1) for every

Received by the editors March 27, 1980 and, in revised form, August 6, 1980.

1980 Mathematics Subject Classification. Primary 34G05.

Key words and phrases. Semigroup of operators, abstract differential equation, weak solution, bounded semivariation.

'Operated by Union Carbide Corporation under contract W-7405-eng-26 with the U.S. Department of Energy. 
continuous function $f$ if and only if the semigroup $T(t), t>0$, is of bounded semivariation.

2. Operators of bounded semivariation. Given a closed interval $[a, b]$ of the real line, a subdivision of $[a, b]$ is a finite sequence $d$ : $a=d_{0}<d_{1}<\cdots<d_{n}=b$. Let $D[a, b]$ denote the set of all subdivisions of $[a, b]$, and $X$ and $Y$ be Banach spaces. For $\alpha:[a, b] \rightarrow L(X ; Y)$ and $d \in D[a, b]$, define

$$
S V_{d}[\alpha]=\sup \left\{\left\|\sum_{i=1}^{n}\left[\alpha\left(d_{i}\right)-\alpha\left(d_{i-1}\right)\right] x_{i}\right\| \mid x_{i} \in X,\left\|x_{i}\right\|<1\right\}
$$

and $S V[\alpha]=\sup \left\{S V_{d}[\alpha] \mid d \in D[a, b]\right\}$. We say $\alpha$ is of bounded semivariation (see [4]) if $S V[\alpha]<\infty$.

It follows from

$$
\begin{aligned}
S V_{d}[\alpha] & =\sup \left\{\left\|\sum_{i=1}^{n}\left[\alpha\left(d_{i}\right)-\alpha\left(d_{i-1}\right)\right] x_{i}\right\| x_{i} \in X,\left\|x_{i}\right\|<1\right\} \\
& \leqslant \sum_{i=1}^{n}\left\|\alpha\left(d_{i}\right)-\alpha\left(d_{i-1}\right)\right\|,
\end{aligned}
$$

that the concept of bounded semivariation is an extension of the concept of bounded variation. For functions, the concepts of bounded semivariation and bounded variation are equivalent. In fact, if $f:[a, b] \rightarrow X \subset L\left(X^{\prime} ; C\right)$ and $\langle\cdot, \cdot\rangle$ is the pairing between $X$ and $X^{\prime}$, then

$$
\begin{aligned}
S V_{d}[f] & =\sup \left\{\left|\sum_{i=1}^{n}\left\langle f\left(d_{i}\right)-f\left(d_{i-1}\right), x_{i}\right\rangle\right| \mid x_{i} \in X^{\prime},\left\|x_{i}\right\|<1\right\} \\
& =\sum_{i=1}^{n}\left\|f\left(d_{i}\right)-f\left(d_{i-1}\right)\right\| .
\end{aligned}
$$

The following propositions are stated without proof.

Proposition 2.1 (HöNIG). If $\alpha:[a, b] \rightarrow L(X ; Y)$ is of bounded semivariation and $f:[a, b] \rightarrow X$ is continuous, then the Riemann-Stieltjes integral $\int_{a}^{b} d \alpha(s) f(s)$ exists.

LEMMA 2.1. If $\alpha:[0, r] \rightarrow L(X ; Y)$ is of bounded semivariation, $\alpha(t)$ is strongly continuous at $t=0$, and $f:[0, r] \rightarrow X$ is continuous, then

$$
\lim _{t \rightarrow 0^{+}} \int_{0}^{t} d \alpha(s) f(s)=0
$$

3. Regularity of solutions. When $F$ is the class of Hölder continuous functions, Crandall and Pazy [3] demonstrate that

(i) $T(t) X \subset D(A)$ for $t>0$,

(ii) $\lim _{t \rightarrow 0^{+}} t^{p}\|A T(t)\|<\infty$ for all $p>1$,

are necessary and sufficient conditions on the semigroup $T(t)$ to assure that the function (1.3) is a strong solution of the Cauchy problem (1.1) for every $f \in F$. Webb [8] establishes $T(t) X \subset D(A)$ for $t>0$ as a sufficient condition for every weak solution to be a strong solution when $F$ is the class of continuous functions of bounded variation. Our principal result will be to establish bounded semivariation as a necessary and sufficient condition on the semigroup $T(t), t>0$, to assure that (1.3) is a strong solution of (1.1) when $F$ is the class of continuous functions. We first establish some necessary lemmas. 
LEMMA 3.1. If $f$ is continuous and $T(\cdot)$ is of bounded semivariation on $[0, r]$, then for $t \in[0, r]$,

$$
\int_{0}^{t} T(t-s) f(s) d s \in D(A)
$$

and

$$
A \int_{0}^{t} T(t-s) f(s) d s=\int_{0}^{t} d_{s} T(t-s) f(s)
$$

Proof. Under the assumptions on $f$ and $T(\cdot)$, it follows from Proposition 2.1 that the Riemann-Stieltjes integral $\int_{0}^{t} d_{s} T(t-s) f(s)$ exists. For a fixed positive integer $n$, let $d_{i}^{n}=i t / n$, where $i=0,1, \ldots, n$. Define $g_{n}(s)=T(t-s) f\left(d_{i}^{n}\right)$ for $d_{i-1}^{n}<s<$ $d_{i}^{n}$ and $g_{n}(0)=T(t) f(0)$. Since the sequence $\left\{g_{n}\right\}$ is uniformly bounded and converges to $T(t-s) f(s)$ on $[0, t]$,

$$
\lim _{n \rightarrow \infty} \int_{0}^{t} g_{n}(s) d s=\int_{0}^{t} T(t-s) f(s) d s .
$$

Since $\int_{0}^{t} g_{n}(s) d s \in D(A)$ and

$$
A \int_{0}^{t} g_{n}(s) d s=\sum_{i=1}^{n}\left[T\left(t-d_{i}^{n}\right)-T\left(t-d_{i-1}^{n}\right)\right] f\left(d_{i}^{n}\right) \rightarrow \int_{0}^{t} d_{s} T(t-s) f(s),
$$

(3.1) and (3.2) follow from the closedness of $A$.

LEMMA 3.2. If $f$ is continuous and $T(\cdot)$ is of bounded semivariation on $[0, r]$, then $\int_{0}^{t} d_{s} T(t-s) f(s)$ is continuous in $t$ on $[0, r]$.

Proof. Assume $0<t<r$ and $0<\Delta t$, and observe that

$$
\begin{aligned}
\int_{0}^{t+\Delta t} d_{s} T(t+\Delta t-s) f(s)-\int_{0}^{t} d_{s} T(t-s) f(s) \\
=[T(\Delta t)-I] \int_{0}^{t} d_{s} T(t-s) f(s)+\int_{0}^{\Delta t} d_{s} T(t-s) f(t+\Delta t-s) .
\end{aligned}
$$

Since it follows from Lemma 2.1 that $\lim _{\Delta t \rightarrow 0^{+}} \int_{0}^{\Delta t} d_{s} T(t-s) f(t+\Delta t-s)=0$, continuity of $\int_{0}^{t} d_{s} T(t-s) f(s)$ from the right is a consequence of the fact that $\lim _{h \rightarrow 0^{+}}[T(h)-I] x=0$ for all $x \in X$.

To show continuity from the left, let $0<t<r$ and $0<\Delta t$. Then

$$
\begin{aligned}
\int_{0}^{t} d_{s} T(t-s) & f(s)-\int_{0}^{t-\Delta t} d_{s} T(t-\Delta t-s) f(s) \\
& =\int_{0}^{t-\Delta t} d_{s} T(t-\Delta t-s)(T(\Delta t)-I) f(s)+\int_{t-\Delta t}^{t} d_{s} T(t-s) f(s) \\
& =\int_{0}^{t-\Delta t} d_{s} T(s)(T(\Delta t)-I) f(t-\Delta t-s)+\int_{0}^{\Delta t} d_{s} T(s) f(t-s) .
\end{aligned}
$$

Since

$$
\left\|\int_{0}^{t-\Delta t} d_{s} T(s)(T(\Delta t)-I) f(t-\Delta t-s)\right\|<S V[T(\cdot)] \sup _{0<s<t-\Delta t}\|(T(\Delta t)-I) f(s)\|
$$

and $\lim _{\Delta t \rightarrow 0^{+}} \int_{0}^{\Delta t} d_{s} T(s) f(t-s)=0$, continuity from the left follows from the uniform continuity of $f$ on $[0, r]$ and the fact that $\lim _{h \rightarrow 0^{+}}(T(h)-I) z=0$ uniformly for $z$ in a compact subset of $X$. 
Proposition 3.1. The function (1.3) is a strong solution of the Cauchy problem (1.1) for every continuous function $f$ if and only if $T(\cdot)$ is of bounded semivariation on $[0, r]$.

Proof. It is easily shown (Pazy [6]) that the weak solution $v$ defined by (1.3) is a strong solution of (1.1) if and only if $v(t) \in D(A)$ and $A v(t)$ is continuous on [0,r]. If $f$ is continuous and $T(\cdot)$ of bounded semivariation on $[0, r]$, then these conditions are satisfied by virtue of Lemmas 3.1 and 3.2.

To demonstrate the necessity of the assumption that $T(\cdot)$ be of bounded semivariation on $[0, r]$, define the bounded linear operator

$$
L: C([0, r] ; X) \rightarrow X \text { by } L(f)=\int_{0}^{r} T(r-s) f(s) d s .
$$

Since every weak solution is a strong solution, $L(f) \in D(A)$ for every continuous $f$. It follows from the closedness of $A$ and the boundedness of $L$ that $A L$ : $C([0, r] ; X) \rightarrow X$ is bounded. Let $\left\{d_{i}\right\}$ be a subdivision of $[0, r]$ and $\varepsilon$ a positive real number less than $\min \left\{\left|d_{i}-d_{i-1}\right|\right\}$. For every fixed but arbitrary sequence $x_{i} \in X$, $\left\|x_{i}\right\|<1,2, \ldots, n+1$, define the continuous function $f_{d, e}:[0, r] \rightarrow X$ by

$$
f_{d, \varepsilon}(\tau)= \begin{cases}x_{i} & \text { if } d_{i-1}<\tau<d_{i}-\varepsilon, \\ x_{i+1}+\left(x_{i+1}-x_{i}\right)\left(\tau-d_{i}\right) / \varepsilon & \text { if } d_{i}-\varepsilon<\tau<d_{i} .\end{cases}
$$

Using the fact that

$$
A \int_{a}^{b} s T(r-s) x d s=\int_{a}^{b} T(r-s) x d s-b T(r-b) x+a T(r-a) x,
$$

we obtain

$$
\begin{aligned}
A L\left(f_{d, \varepsilon}\right)= & A \int_{0}^{r} T(r-s) f_{d, \varepsilon}(s) d s \\
= & A\left[\sum_{i=1}^{n} \int_{d_{i-1}}^{d_{i}-\varepsilon} T(r-s) f_{d, \varepsilon}(s) d s+\int_{d_{i}-\varepsilon}^{d_{i}} T(r-s) f_{d, \varepsilon}(s) d s\right] \\
= & A\left[\sum_{i=1}^{n} \int_{d_{i-1}}^{d_{i}-\varepsilon} T(r-s) x_{i} d s\right. \\
& \left.+\int_{d_{i}-\varepsilon}^{d_{i}} T(r-s)\left(x_{i+1}+\left(x_{i+1}-x_{i}\right)\left(s-d_{i}\right) / \varepsilon\right) d s\right] \\
= & \sum_{i=1}^{n}\left[-T\left(r-d_{i}+\varepsilon\right)+T\left(r-d_{i-1}\right)\right] x_{i} \\
& +\sum_{i=1}^{n}\left[-T\left(r-d_{i}\right)+T\left(r-d_{i}+\varepsilon\right)\right] x_{i+1} \\
& +\sum_{i=1}^{n} \frac{1}{\varepsilon} \int_{d_{i}-\varepsilon}^{d_{i}} T(r-s)\left(x_{i+1}-x_{i}\right) d s-\sum_{i=1}^{n} T\left(r-d_{i}+\varepsilon\right)\left(x_{i+1}-x_{i}\right) \\
= & -\sum_{i=1}^{n}\left[T\left(r-d_{i}\right)-T\left(r-d_{i-1}\right)\right] x_{i}+\sum_{i=1}^{n} \frac{1}{\varepsilon} \int_{d_{i}-\varepsilon}^{d_{i}} T(r-s)\left(x_{i+1}-x_{i}\right) d s \\
& -\sum_{i=1}^{n} T\left(r-d_{i}\right)\left(x_{i+1}-x_{i}\right) .
\end{aligned}
$$


Thus

$$
\begin{aligned}
\left\|\sum_{i=1}^{n}\left[T\left(r-d_{i}\right)-T\left(r-d_{i-1}\right)\right] x_{i}\right\| \\
\quad<\|A L\|+\sum_{i=1}^{n}\left\|\frac{1}{\varepsilon} \int_{d_{i}-\varepsilon}^{d_{i}} T(r-s)\left(x_{i+1}-x_{i}\right) d s-T\left(r-d_{i}\right)\left(x_{i+1}-x_{i}\right)\right\| .
\end{aligned}
$$

Taking the limit as $\varepsilon \rightarrow 0$, we obtain that $\left\|\sum_{i=1}^{n}\left[T\left(r-d_{i}\right)-T\left(r-d_{i-1}\right)\right] x_{i}\right\|<$ $\|A L\|$. It follows that $S V[T(\cdot)]<\|A L\|$ and the proof is complete.

ExAMPLE. The semigroup $T(\cdot): c_{0} \rightarrow c_{0}$ defined by $T(t) X=\left(e^{-n t} x_{n}\right)$ is an example of a strongly continuous semigroup of bounded semivariation with an unbounded infinitesimal generator.

The characterization of strongly continuous semigroups of bounded semivariation remains an open question. However, we can establish some partial results in this direction.

LEMMA 3.3. A necessary condition for a strongly continuous semigroup $T(\cdot)$ to be of bounded semivariation on $[0, r]$ is that $T(t)$ be analytic.

Proof. It suffices to prove that $t A T(t)$ is bounded for $t \in[0, r]$ (see Pazy [6, p. 61]). For an arbitrary but fixed $x \in X$ define $f(t)=T(t) x$ and consider the Cauchy problem $u^{\prime}(t)=A u(t)+f(t), u(0)=0$. The unique weak solution of this equation is given by $u(t)=t T(t) x$. By Proposition 3.1, this solution is a strong solution and consequently $A u(t)=t A T(t) x$ is continuous on $[0, r]$, establishing the result.

LEMMA 3.4. A necessary condition for a strongly continuous semigroup $T(t), t>0$, of bounded semivariation on $X$ to have an unbounded infinitesimal generator is that $X$ contain a subspace isomorphic to $c_{0}$.

Proof. If $T(t), t \geqslant 0$, is of bounded semivariation then $A L: C([0, r] ; X) \rightarrow X$ is bounded, where $L$ is defined by (3.5). The proof follows from the fact that a strongly continuous semigroup $T(t), t>0$, defined on a Banach space $X$, not containing $c_{0}$, has a bounded infinitesimal generator $A$ if and only if $A L$ : $C([0, r] ; X) \rightarrow X$ is bounded [1].

REMARK. The Banach spaces $c_{0}, l^{\infty}, L^{\infty}$, and $C$ contain copies of $c_{0}$, while any reflexive space, $L^{1}$ and $l^{1}$ do not.

COROLlary 3.1. In a reflexive Banach space, a weak solution of (1.1) is a strong solution for every continuous function $f$ if and only if $A$ is bounded.

LEMMA 3.5. A strongly continuous group is of bounded semivariation if and only if its infinitesimal generator is bounded.

Proof. The proof follows from the fact that analytic groups have bounded generators.

\section{REFERENCES}

1. J. B. Baillon, Caractère bone' de certains générateurs de semigroupes linéaire dans les espaces de Banach (to appear). 
2. J. M. Ball, Strongly continuous semigroups, weak solutions, and the variation of constants formula, Proc. Amer. Math. Soc. 63 (1977), 370-373.

3. M. G. Crandall and A. Pazy, On the differentiability of weak solutions of a differential equation in Banach space, J. Math. Mech. 18 (1969), 1007-1016.

4. C. S. Hönig, The Green function of a linear differential equation with a lateral condition, Bull. Amer. Math. Soc. 79 (1973), 587-593.

5. T. Kato, Perturbation theory for linear operators, Springer-Verlag, New York, 1966.

6. A. Pazy, Semi-groups of linear operators and applications to partial differential equations, Lecture Notes \# 10, University of Maryland, 1974.

7. R. S. Phillips, Perturbation theory for semi-group of linear operators, Trans. Amer. Math. Soc. 74 (1953), 199-221.

8. G. F. Webb, Regularity of solutions to an abstract inhomogeneous linear differential equation, Proc. Amer. Math. Soc. 62 (1977), 271-277.

Technology Assessments Section, Health and Safety Research Division, OAk Ridge National Laboratory, OAK RIDGe, Tennessee 37830 